\title{
ELF wave generation in the ionosphere using pulse modulated HF heating: initial tests of a technique for increasing ELF wave generation efficiency
}

\author{
R. Barr ${ }^{1}$, P. Stubbe ${ }^{2}$, M. T. Rietveld ${ }^{3}$ \\ ${ }^{1}$ National Institute of Water and Atmospheric Research, PO Box 14901, Kilbirnie, Wellington, New Zealand, e-mail: r.barr@niwa.cri.nz \\ ${ }^{2}$ Max-Planck-Institut für Aeronomie, D-37189, Katlenburg-Lindau, Germany \\ ${ }^{3}$ EISCAT, Ramfjormoen, N-9020, Tromsdalen, Norway
}

Received: 9 July 1998 / Revised: 16 November 1998 / Accepted: 20 November 1998

\begin{abstract}
This paper describes the results of a preliminary study to determine the effective heating and cooling time constants of ionospheric currents in a simulated modulated HF heating, 'beam painting' configuration. It has been found that even and odd harmonics of the fundamental ELF wave used to amplitude modulate the HF heater are sourced from different regions of the ionosphere which support significantly different heating and cooling time constants. The fundamental frequency and its odd harmonics are sourced in a region of the ionosphere where the heating and cooling time constants are about equal. The even harmonics on the other hand are sourced from regions of the ionosphere characterised by ratios of cooling to heating time constant greater than ten. It is thought that the even harmonics are sourced in the lower ionosphere (around $65 \mathrm{~km}$ ) where the currents are much smaller than at the higher altitudes around $78 \mathrm{~km}$ where the currents at the fundamental frequency and odd harmonics maximise.
\end{abstract}

Key words. Electromagnetics (antennae) - Ionosphere (active experiments) - Radio science (non linear phenomena)

\section{Introduction}

The geophysical technique for ELF wave generation we present here has its origins in the work of Willis and Davis (1973). They were the first to suggest the possibilty of modulating the ionospheric dynamo current in the Eregion by changing the ionospheric conductivity using powerful, pulsed HF waves. They were mainly interested

Correspondence to: R. Barr in slow ionospheric electron-density changes resulting from the chemical reactions which were electron temperature dependent, the electron temperature having been increased by the pulsed HF heating. Their system was thus aimed at the generation of long period ULF waves. The first reported detection of ELF/VLF signals radiated from the ionosphere, when heated by powerful HF waves modulated at ELF/VLF, was presented by Getmantsev et al. (1974). They detected weak signals over a frequency range $1-7 \mathrm{kHz}, 180 \mathrm{~km}$ north of a $150 \mathrm{~kW}$ HF transmitter amplitude modulated between 1 and $7 \mathrm{kHz}$. The transmitter was located at mid-latitudes. Kotik and Trakhtengerts (1975) correctly explained the signals in terms of the modulation of natural currents in the ionosphere and suggested that stronger signals would be obtained by repeating the experiment at a higher latitude, in a region of stronger natural current flow. Kapustin et al. (1977) may have followed this suggestion, as two years later they reported nocturnal measurements made at higher latitudes, beneath the auroral electrojet. Sadly they only presented relative field strength measurements and therefore it was not possible to estimate from their work whether the signals had indeed increased in amplitude with the move to higher latitudes. Kapustin et al. (1977) did however show how the signals were strongly dependent on the strength of the auroral electrojet.

Stubbe et al. (1982) presented the first calibrated recordings of ELF/VLF waves radiated from the auroral electrojet, when modulated by a powerful amplitude modulated HF transmitter. Working with the $1 \mathrm{MW}$ Tromsø HF heating facility they detected ELF/VLF magnetic flux densities, directly below the heated patch, peaking around $1 \mathrm{pT}$. The first long-range detection of ELF/VLF signals from the heated ionosphere was made by Barr et al. (1985) at Lycksele, $500 \mathrm{~km}$ south of the Tromsø heater. The signals were weak, $\sim 30$ femto Tesla and as such were in agreement with the theoretical predictions of Barr and Stubbe (1984a), who predicted the Tromsø facility would produce radiated powers increasing from $\sim 1 \mathrm{~mW}$ to $1 \mathrm{~W}$ over the frequency range 
$100 \mathrm{~Hz}$ to $3 \mathrm{KHz}$. When one considers the HF power output at Tromsø is $\sim 1 \mathrm{MW}$ one can see that the basic 'Polar Electrojet Antenna' system (PEJ), as it was initially described, was not an efficient ELF radiator when compared for example with the horizontal electric dipole (Burrows, 1978). Techniques to increase the efficiency of ELF wave generation by the Polar Electrojet Antenna were proposed by Papadopoulos et al. (1990). The work presented here describes our first attempts at Tromsø to test some aspects of one of these techniques, the so called 'beam painting' technique.

\section{Experimental Technique}

\subsection{Beam painting}

The beam painting technique of Papadopoulos et al. (1989) relies for its operation on the fact that, in general, the electron heating and cooling time constants in the ionosphere are not equal, and that over a large range of ionospheric heights the heating time constant may in fact be considerably smaller than the cooling time constant (see Rietveld et al., 1986). Together with this special ionospheric environment, the beam painting technique requires a high power HF transmitter, the antenna of which has a very narrow beamwidth (Papadopoulos et al., 1989). With such a system the procedure is to deflect the intense, narrow HF beam over a range of spots in the ionosphere within the total deflection range of the antenna (see Fig. 1). The idea is to heat each individual spot in the ionosphere for a time of the order of the electron heating time constant and, having heated every spot in turn, hope to return to the first spot again before the electrons which were first heated have cooled significantly. That is, return in a time short compared with the electron cooling time constant. To be able to visit a number of spots with such a system, and thus markedly enhance the ELF generation efficiency of the

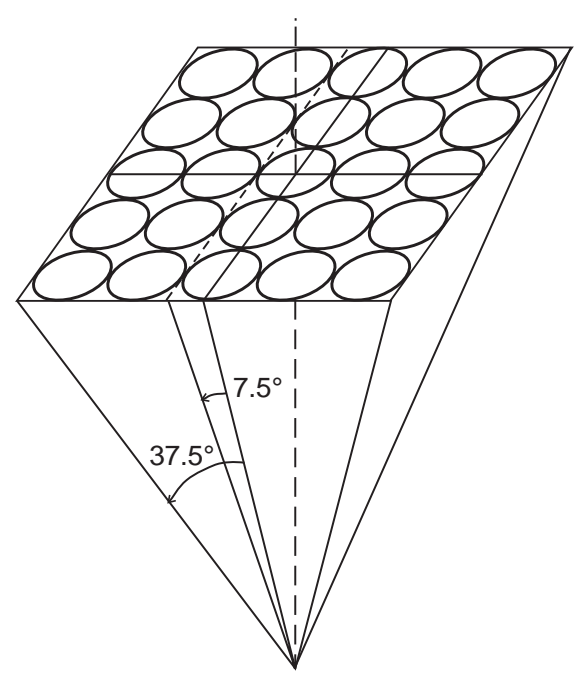

Fig. 1. Technique for enhancing ELF radiated power by scanning the $\mathrm{HF}$ beam over an area within the antenna beam angle of $\pm 37.5^{\circ}$ (see Papadopoulos et al., 1989)
PEJ, it is necessary for the electron heating time constant to be significantly shorter than the cooling time constant (Papadopoulos et al., 1989). The technique we describe below is an initial attempt to approximate to the beam painting technique in order to try and determine effective heating and cooling time constant values directly applicable to beam painting.

\subsection{Transmitter configuration}

The data presented here were obtained between 10:46 and 12:22 UT on 7 September 1990. Throughout this interval the Tromsø facility was operating on $4.04 \mathrm{MHz}$ with $960 \mathrm{~kW}$ of power into antenna field 2 $(3.85-5.65 \mathrm{MHz})$. The transmitter was switched to radiate ' $\mathrm{X}$ ' and ' $\mathrm{O}$ ' mode polarisation on alternate 4min periods (the ' $\mathrm{X}$ ' mode periods starting on the hour). Similar configurations have been used in many previous experiments. The feature unique to this particular experiment was the amplitude modulation applied to the transmitter.

It was initially thought that a direct test of the beam painting technique might be feasible using a beam deflection facility on the Tromsø transmitter (see Rietveld et al., 1984, 1993), but this input had a limited bandwidth of $5 \mathrm{kHz}$. The transmitter facility was however equipped with two very fast amplitude modulation ('keying') inputs whose bandwidths were measured in hundreds of $\mathrm{kHz}$. (These two high speed inputs, whose outputs were logically ANDed to produce the amplitude modulation, were required in early experiments with the Tromsø facility when it was used in conjunction with the SOUSY HF radar). It was therefore decided to attempt to simulate a beam painting study using high speed pulsed amplitude modulation as described below. It should be noted that the experimental configuration to be described was designed to simulate the effects of electron heating and cooling time constants on the performance of a beam painting heating facility and not the signal enhancements due to spatial separation of mutiple heated patches of ionosphere.

This experiment was specifically designed to simulate the effects of using beam painting to produce a $505 \mathrm{~Hz}$ ELF signal. Figure 2 shows on its upper trace the fundamental period $505 \mathrm{~Hz}$ square wave which was applied to the first of the SOUSY inputs on the heater. The three lower traces show examples of the pulse waveforms which were applied to the second SOUSY input. The second input was derived from a $10.1 \mathrm{kHz}$ signal locked to the 20th harmonic of the $505 \mathrm{~Hz}$ signal. The main feature of the $10.1 \mathrm{kHz}$ signal was that its duty cycle could be varied form 0 to $100 \%$. The three lower traces in Fig. 2 show examples with duty cycles of 10,50 and $90 \%$.

The three lower traces in Fig. 3 show the result of ANDing the three lower traces of Fig. 2 with the upper trace in Fig. 2. As such the three lower traces in Fig. 3 represent the resultant modulation applied to the heater transmitter, and hence the HF output of the transmitter, for duty cycles of 10,50 and $90 \%$ of the $10.1 \mathrm{kHz}$ 

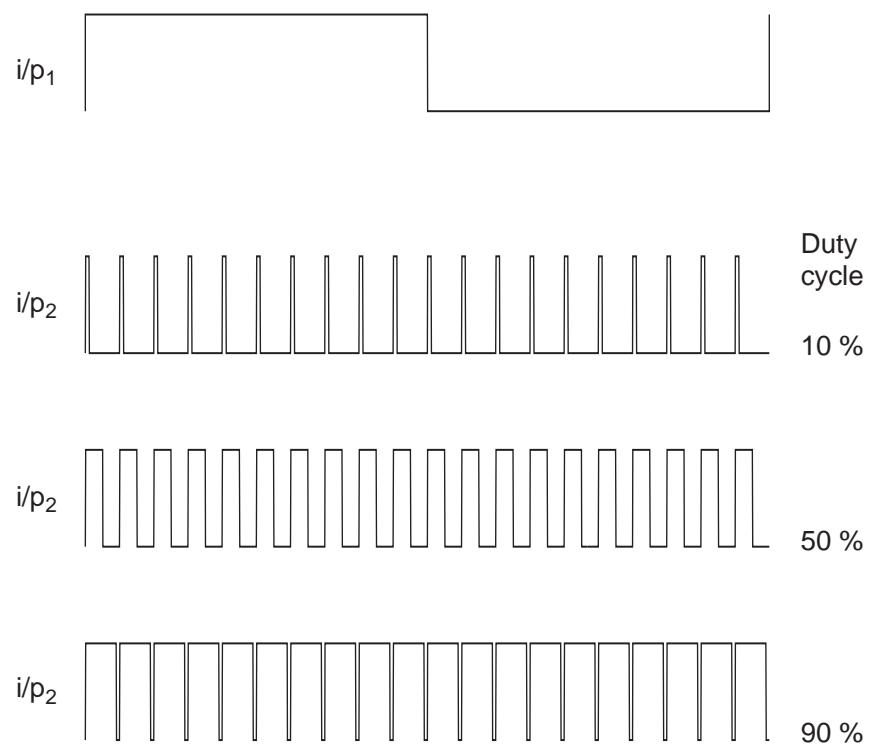

Fig. 2. "Sousy switch" gate input waveforms. $i / p_{1}$ - fundamental ELF waveform, typically $505 \mathrm{~Hz} . i / p_{2}$ - variable duty cycle waveform $(0-100 \%)$, typically $10.1 \mathrm{kHz}$

waveform. With a duty cycle of $0 \%$ the heater was completely 'off' and with a duty cycle of $100 \%$ the heater was 'on' and fully amplitude modulated at $505 \mathrm{~Hz}$.

\subsection{Rationale for modulation waveform}

The idea behind the modulation waveform used in our experiment is hinted at in the three lower traces in Fig. 3. These traces represent the pulsed HF output of the transmitter and hence also a lower limit to the power

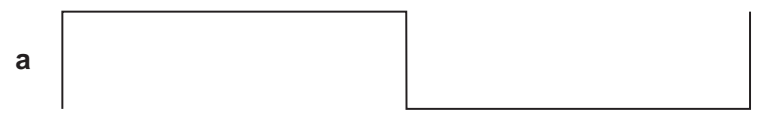

b

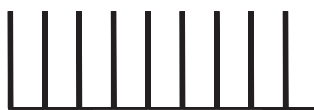

c

|l||l||l||

d

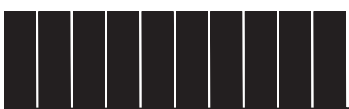

Duty cycle $10 \%$

$50 \%$

$90 \%$

Fig. 3. HF heater transmitter keying waveform. a desired ELF current in ionosphere, b-d effective HF output from heater transmitter with pulsed amplitude modulation of 10,50 and $90 \%$ duty cycle respectively input to the heater transmitter. As an example, assume the electrons in the ionosphere are efficiently heated in the time of the narrow pulses presented in the waveform for the $10 \%$ duty cycle, but do not cool markedly in a time some ten times greater than this. Then the effective electron heating by the waveform of $10 \%$ duty cycle may be expected to approach the heating produced by a 90 or $100 \%$ waveform whilst consuming only one tenth the input power. To check this belief we computed the ionospheric response to various pulsed $\mathrm{HF}$ heating regimes, similar to those displayed in Fig. 3. We assume that the ELF/VLF currents vary exponentially with HF heating, with heating time constant, $\tau_{h}$, and cooling time constant, $\tau_{c}$ (see Rietveld et al., 1986).

The upper panel in Fig. 4 shows the ELF pulse response to heating with $10 \%$ and $100 \%$ duty cycle waveforms when the heating and cooling time constants in the ionosphere are both assumed to be $10 \mu \mathrm{s}$. The lower panel shows the response to the same HF pulse waveforms but with the cooling time constant of the ionospheric currents now increased to $300 \mu$ s. It can be seen from Fig. 4 that the efficiency of ELF wave generation with heating of low duty cycle $(10 \%)$ is greatly enhanced when the ionospheric cooling time constant is greater than the heating time constant. To show this more directly waveforms similar to those in Fig. 4 were Fourier transformed to ascertain the spectral content of the composite waveforms at the fundamental frequency of $505 \mathrm{~Hz}$.

Figure 5 shows the spectral amplitude at $505 \mathrm{~Hz}$, as a function of duty cycle, produced by heating using the pulse waveforms of Fig. 3. The six separate curves represent the result of computations with different ionospheric cooling time constants ranging from $1-300 \mu \mathrm{s}$ and a constant ionospheric heating time constant of $10 \mu$ s. The thick solid line, which shows a linear spectral increase with duty cycle from $0-100 \%$, represents the case where the ionospheric heating and cooling time constants were each equal to $10 \mu \mathrm{s}$. It is immediately clear from Fig. 5 that if the heating time constant for the ionospheric currents is much less than the cooling time constant then significant efficiency advantages can be gained by heating using duty cycles less than $100 \%$. To show this more clearly we present normalised spectra in Fig. 6. These are amplitude spectra, similar to those in Fig. 5, but normalised to the effective transmitter input power, the amplitude response having been divided by the duty cycle. The data in Fig. 6 thus allow one to compare the ELF generation efficiency using a range of duty cycles under differing ionospheric conditions.

The two panels in Fig. 6 show data computed for heating time constants, $\tau_{h}$, of 3 and $100 \mu \mathrm{s}$. The six curves in each panel indicate how the normalized ELF response varies with the duty cycle of the pulse waveform for ionospheric cooling time constants varying from $0.1 \tau_{h}$ to $30 \tau_{h}$. This range of time constants is similar to that calculated for the Hall current in the Dregion of the ionosphere (Rietveld et al., 1986) and the Hall current is normally regarded as the dominant current producing the ELF/VLF magnetic field signatures at the Earth's surface (Barr and Stubbe, 1984b). 

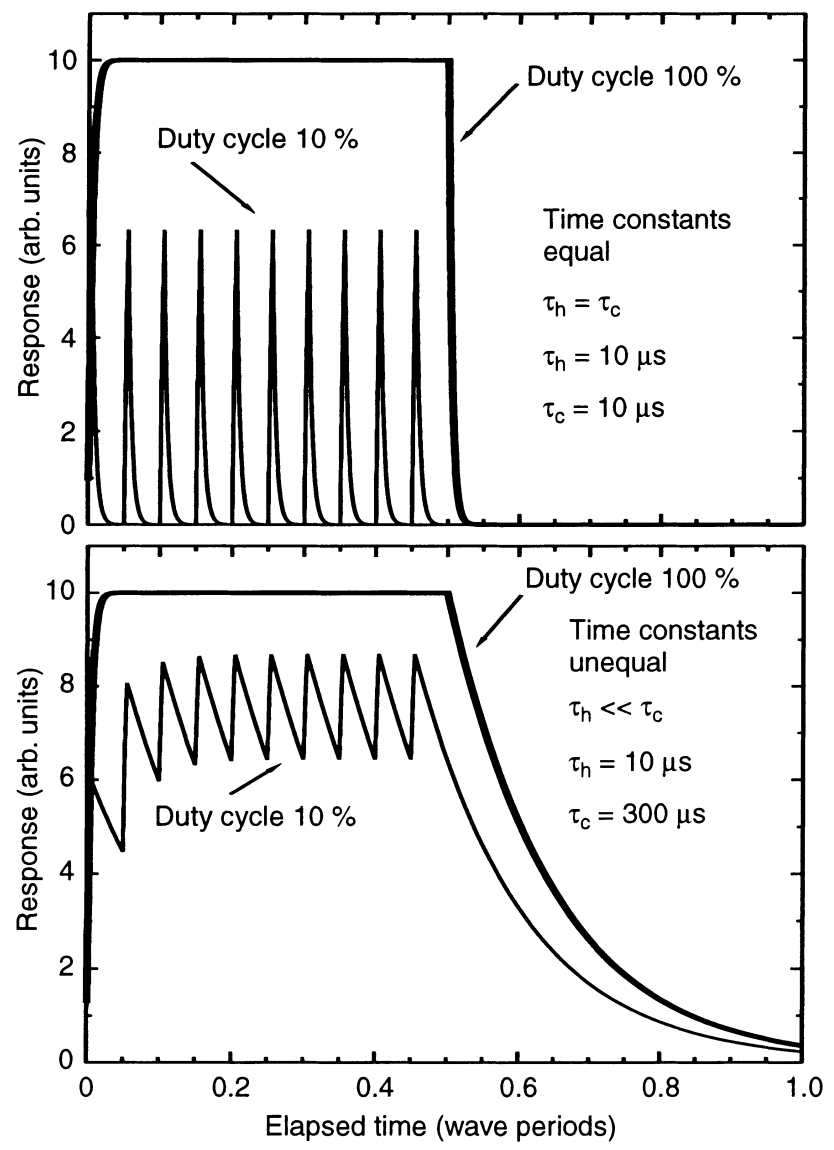

Fig. 4. ELF pulse response of ionosphere to heating with waveforms of 10 and $100 \%$ duty cycle. Upper panel: heating and cooling time constants of ionospheric current both $=10 \mu \mathrm{s}$. Lower panel: heating time constant $=10 \mu \mathrm{s}$. Cooling time constant $=300 \mu \mathrm{s}$

The response axes in Fig. 6 have been scaled so that an ionosphere supporting equal heating and cooling time constants gives a unit response.

It is clear from Fig. 6 that by varying the duty cycle (of the pulse modulation scheme described by Fig. 3) and observing the ionospheric response it should be possible to determine the ratio of the heating to cooling time constants of the dominant ELF/VLF ionospheric current source unambiguously. It is also apparent that it should be possible, with somewhat greater uncertainty, to determine the individual heating and cooling time constants, so long as they are not equal. This was the rationale behind the choice of the modulation waveforms described in sect. 2.2 above. Figure 7 shows a similar set of calculations but evaluated for $9595 \mathrm{~Hz}$, the 19th harmonic of the fundamental frequency of $505 \mathrm{~Hz}$. It is clear that the harmonic data can also be used to determine the ratio of the ionospheric heating and cooling time constants. However it is also clear that independent heating and cooling time constants will only be able to be derived if the heating and cooling time constants differ.

This complex modulation scheme also has the advantage of showing directly how low duty cycle pulsed heating can produce more efficient ELF wave generation than simple square wave modulation, if

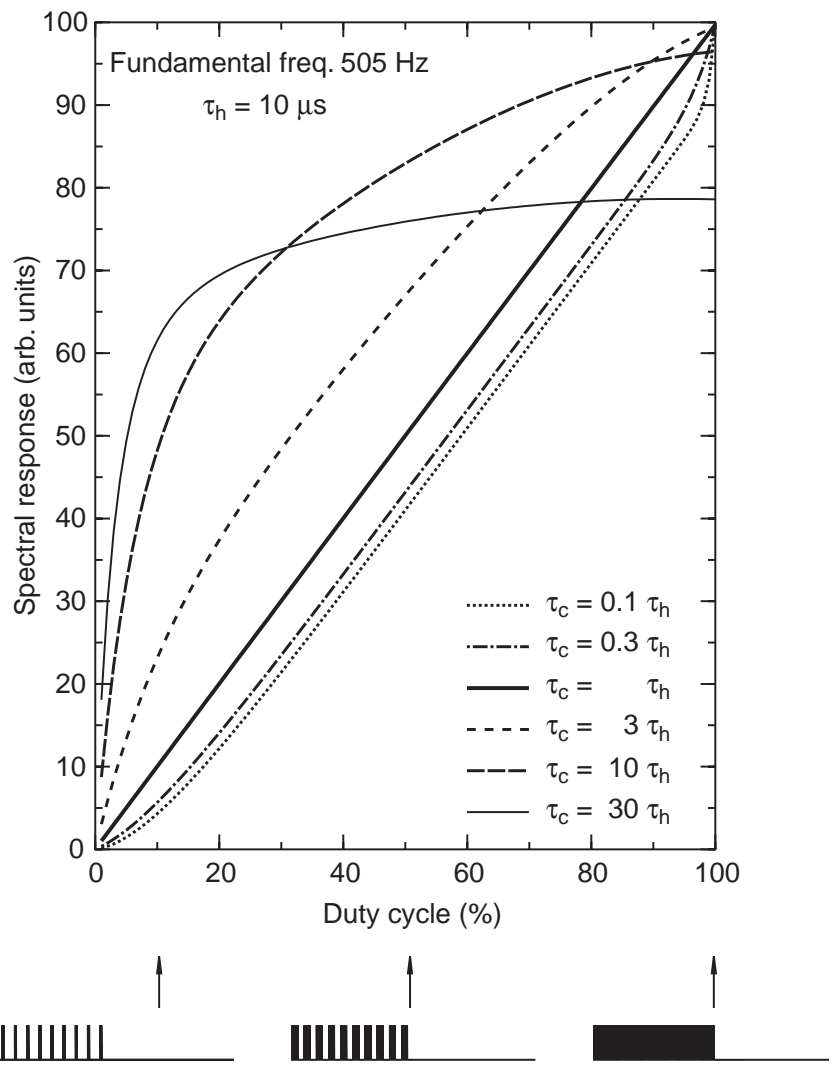

Fig. 5. Spectral response of ionospheric currents to a heating waveform of $505 \mathrm{~Hz}$, pulse modulated with varying duty cycle $(0-$ $100 \%)$

ionospheric heating time constants are much smaller than cooling time constants. For example the upper panels in Fig. 6 show that if the cooling time constant is 30 times larger than the heating time constant then it is about 20 times more efficient to use a pulse wave form, as shown in Fig. 3, with 1\% duty cycle for modulating a heater transmitter, than it is to use the standard square wave modulation. It should be noted, however, that this remarkable enhancement only appears for small ionospheric heating time constants $(\leq 10 \mu \mathrm{s})$. With a $30 \mu \mathrm{s}$ heating time constant for the ionosphere the enhancement drops to about ten times and drops further to only three times with a $100 \mu$ s heating time constant. However, when one is talking about facilities with a $1 \mathrm{MW}$ output power a three times improvement in efficiency is quite important.

\section{ELF/VLF receiving equipment}

The ELF/VLF receivers used for this study were located at the 'Oststation' about $15-\mathrm{km}$ east of the heating facility, at Ramfjordmoen, near Tromsø in Northern Norway. Orthogonal loop antennas were used to pick up the ELF/VLF signals, oriented so that their planes were aligned along the magnetic North-South (NS) and East-West (EW) directions. The antennas were 24-turn triangular loop antennas of height $4 \mathrm{~m}$ and base $8 \mathrm{~m}$ with a DC resistance of 43 ohms. The loop antennas 

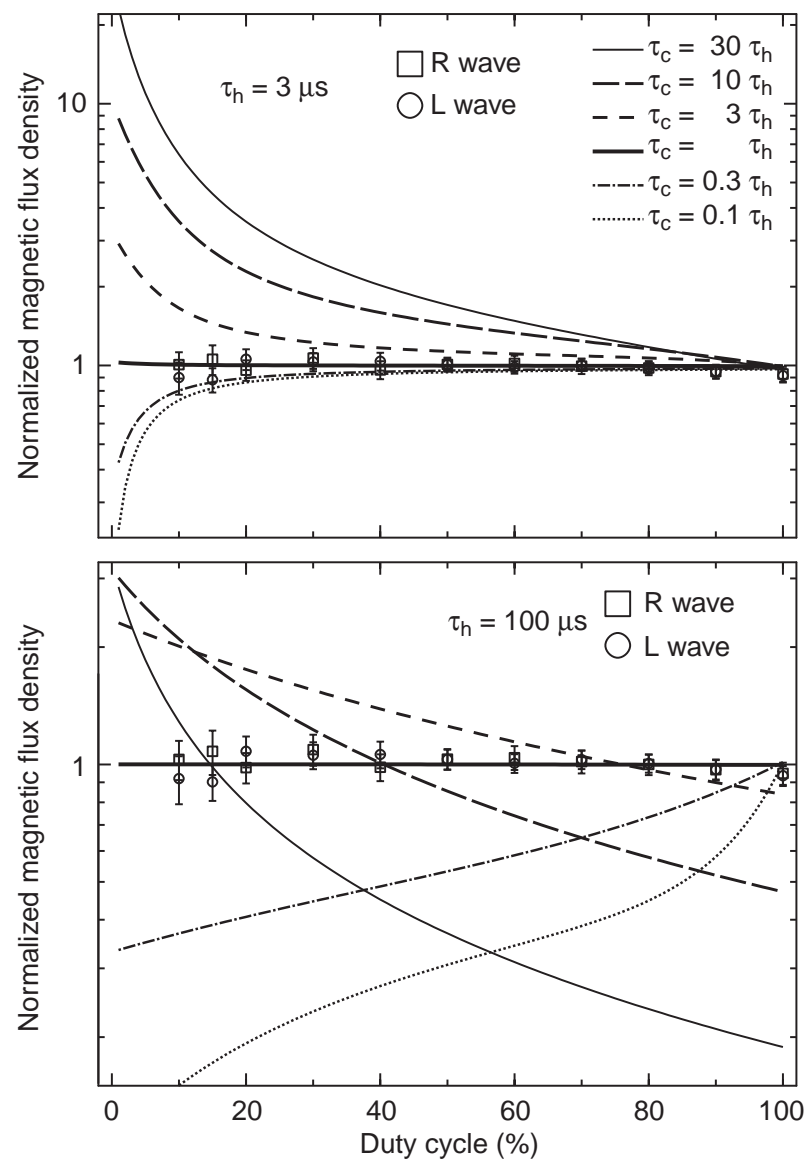

Fig. 6. Theoretical estimates of normalised magnetic flux density at $505 \mathrm{~Hz}$ produced by modulated ionospheric currents, plotted for a range of six cooling time constants. Upper panel data computed for a heating time constant of $3 \mu \mathrm{s}$. Lower panel data computed for a heating time constant of $100 \mu \mathrm{s}$. The square and round markers, with their associated error bars, represent the experimentally measured ' $R$ ' and ' $L$ ' wave normalised magnetic flux densities respectively

were connected to low noise pre-amplifiers the outputs of which were transformer coupled to a Sony digital audio processor (PCM-501ES). This device digitized the signals from the two channels to 16 bit resolution, at a $44 \mathrm{kHz}$ digitization rate, and then serialized the data and coded it onto a video carrier so that it could be permanently recorded on a standard PAL VHS video tape. It was possible to record for $8 \mathrm{~h}$ using a long play video tape, running at half normal speed, which equates with a storage of more than 5 gigabytes of data.

In early reports of ELF/VLF field strength measurements made using the heater facility near Tromsø it was generally the policy to simply present the ELF/VLF signals as directly recorded on the N-S and E-W antennas (Rietveld et al., 1983, 1986; Barr and Stubbe, 1991, 1993). However we can gain more insight into the physical processes taking place in the ionosphere if we convert the N-S and E-W magnetic field components, as recorded at the ground, into the magnetic fields of the right (R) and left (L) handed circularly polarised characteristic waves (Rietveld et al., 1984, 1989; Barr et al., 1997). At the high magnetic latitude of Tromsø the Earth's magnetic field can be assumed to be vertical.
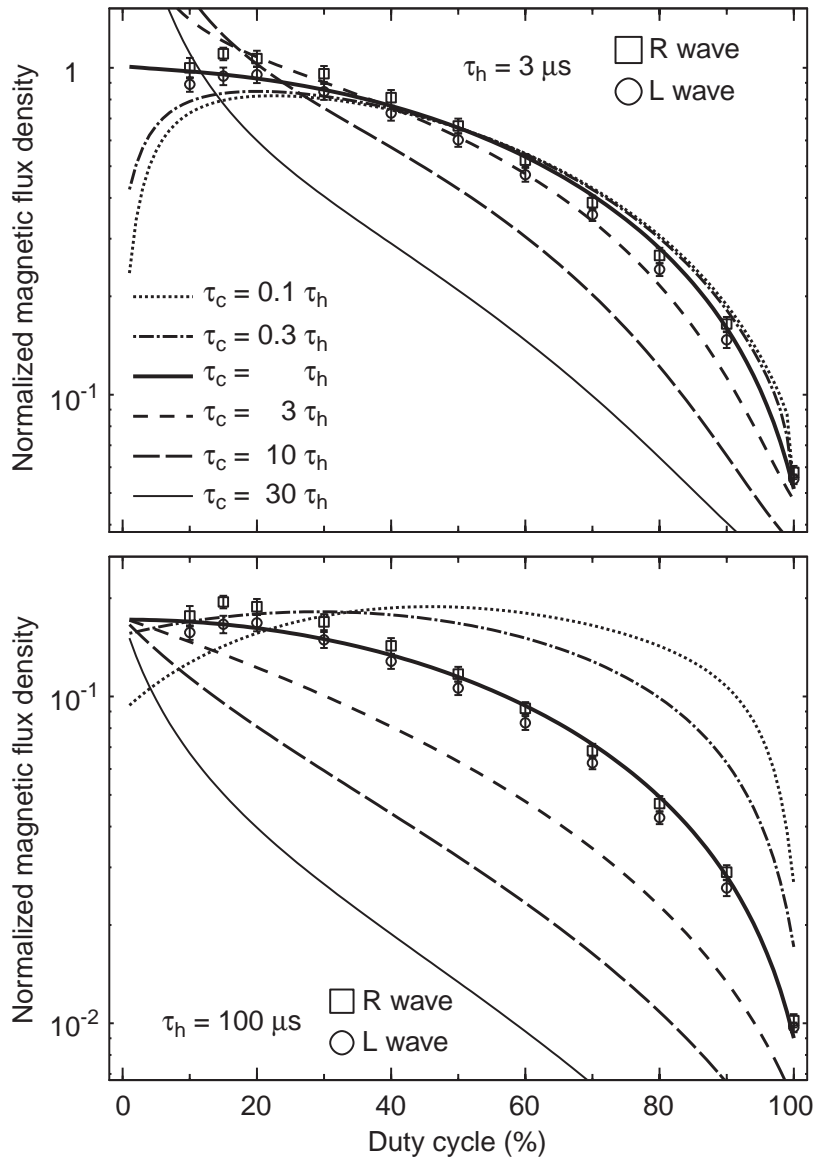

Fig. 7. Theoretical estimates of normalised magnetic flux density at $9595 \mathrm{~Hz}$ produced by modulated ionospheric currents, plotted for a range of six cooling time constants. Upper panel data computed for a heating time constant of $3 \mu \mathrm{s}$. Lower panel data computed for a heating time constant of $100 \mu \mathrm{s}$. The square and round markers, with their associated error bars, represent the experimentally measured ' $R$ ' and ' $L$ ' wave normalised magnetic flux densities respectively

Also the experimental geometry used here implies waves normally incident on the ionosphere. We can therefore assume that the $\mathrm{R}$ and $\mathrm{L}$ circularly polarised modes propagate independently (Budden, 1966). All the ELF/ VLF magnetic field data presented in this paper are therefore displayed in terms of right and left hand characteristic modes.

\section{Results}

\subsection{Odd harmonics}

The ELF/VLF data presented here were recorded between 10:46UT and 12:22UT on 7 September 1990. The transmitter was configured, as described in sect. 2.2, to radiate alternate 4-min segments on ' $\mathrm{X}$ ' and ' $\mathrm{O}$ ' mode polarization. Each 2-min transmission segment was itself subdivided into twelve 10-s segments. During the whole of the transmission period a $505 \mathrm{~Hz}$ square wave was applied to the first SOUSY input and a $10.1 \mathrm{kHz}$ rectangular wave to the second SOUSY input (see sect. 2.2). The duty cycle of the $10.1 \mathrm{kHz}$ waveform was 
changed every $10 \mathrm{~s}$ through the repeating cycle $0,10,15$, $20,30,40,50,60,70,80,90,100 \%$. The upper panel in Fig. 8 shows the magnetic field of the $\mathrm{R}$ wave at the fundamental frequency of $505 \mathrm{~Hz}$ recorded at the Oststation between 11:38 UT and 12:22 UT when the electrojet conditions were reasonably stable. The lower panel shows the signal recorded at $2.525 \mathrm{kHz}$, the 5 th harmonic of the $505 \mathrm{~Hz}$ fundamental.

The data presented in the upper and lower panels of Fig. 8 are very similar, both showing a periodic gradual increase in signal strength with increasing duty cycle of the modulation waveform. Also both the fundamental and harmonic frequencies show preferential signal generation during ' $\mathrm{X}$ ' as opposed to ' $\mathrm{O}$ ' mode heating as reported in most previous studies (Barr et al., 1997; McCarrick et al., 1990; Stubbe et al., 1981).

The $505 \mathrm{~Hz}$ spectral data, averaged for the 11 ' $\mathrm{X}$ ' mode scans shown in Fig. 8, is shown re-plotted in Fig. 6 together with the theoretical data. Data are presented for both the $\mathrm{R}$ and $\mathrm{L}$ mode waves and the mean value of the spectral component is plotted with error bars giving an estimate of the standard deviation of the mean value. It is immediately obvious that the
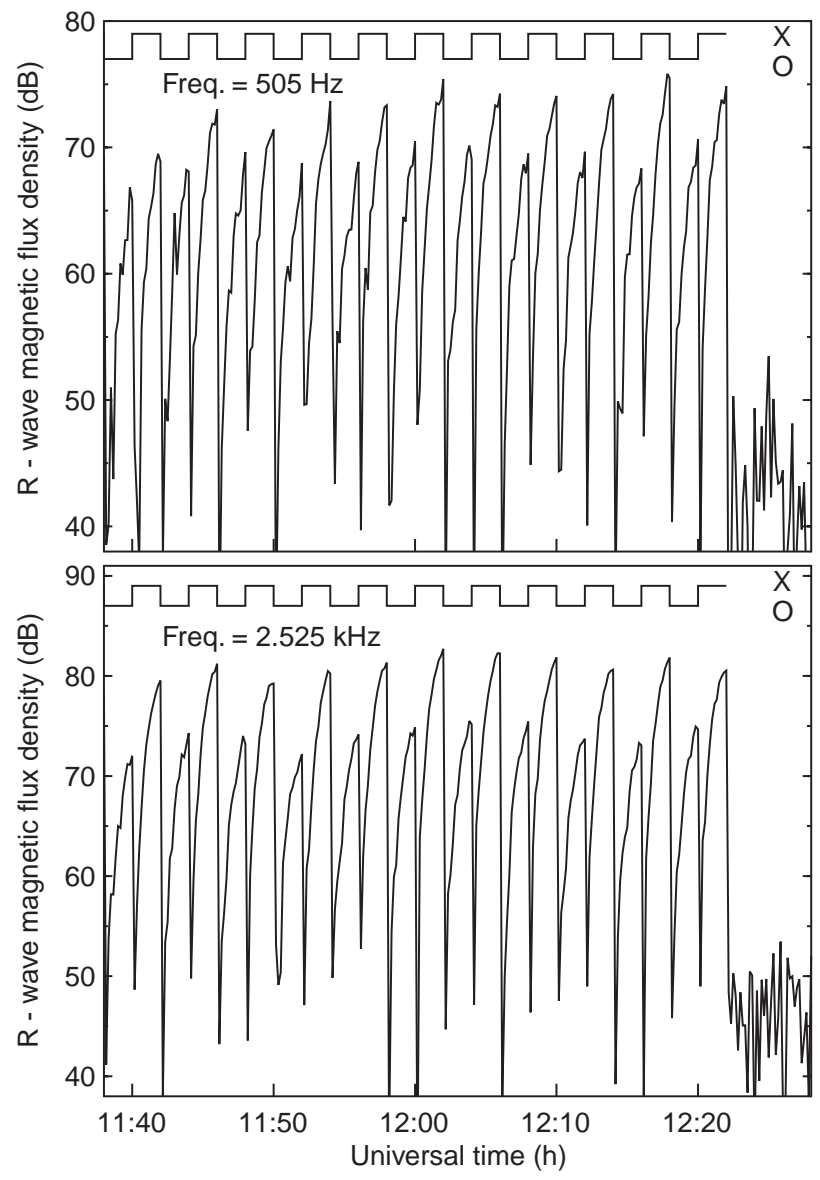

Fig. 8. 'R' wave magnetic flux density as measured at the Oststation. Upper panel: Measurements at the fundamental frequency of $505 \mathrm{~Hz}$. Lower panel: Measurements at the harmonic frequency of $2525 \mathrm{~Hz}$. The crenellated waveform at the top of each panel shows when the heater transmitter was switched between ' $\mathrm{X}$ ' and ' $\mathrm{O}$ ' mode transmissions
$505 \mathrm{~Hz}$ signal is sourced in the ionosphere in a region where the heating and cooling time constants are equal. The $9595 \mathrm{~Hz}$ spectral data (averaged for the $11^{~ ' ~} \mathrm{X}$ ' mode scans shown in Fig. 8) is shown re-plotted on Fig. 7, together with the theoretical data. The data at this high harmonic frequency (19th) suggest that all the odd harmonics generated by pulsed heating, at least during the daytime, are sourced at an altitude where the ionospheric heating and cooling time constants are equal. However whilst this information is useful, if somewhat disappointing with regard to techniques for ELF wave generation efficiency enhancement, it suggests we cannot determine the absolute magnitude of the time constants.

This is confirmed in Fig. 9 where we have plotted coloured contours of the magnitude of the error in least squares fitting the experimental ELF spectral data to theoretically computed spectra. The computations cover ionospheric models with values of heating and cooling time contants each ranging over three orders of magnitude. The program used to minimize the least squares error was a standard National Algorithms group (NAg) minimization subroutine, E04GCF. This program was used to determine the arbitrary scaling factor which provided the minimum error between theoretical computations and the experimental data. This minimum error was then evaluated as a function of the heating and cooling time constants to produce Fig. 9 .

The upper panel of Fig. 9 applies to the spectral data at the $505 \mathrm{~Hz}$ fundamental. The lower panel applies to data at the 13th harmonic, i.e. $6565 \mathrm{~Hz}$. However these two plots are typical of all the odd harmonics for which measurements have been made $(505 \mathrm{~Hz}$ to $9595 \mathrm{~Hz})$. It can be seen that neither plot shows a localised minimum in the least squares error when fitting the experimental data to theory. The data suggest that the absolute magnitude of the heating and cooling time constants cannot be determined to better than an order of magnitude. The data also show however that we can clearly evaluate the ratio of the heating and cooling time constants using the odd harmonic data. Figure 10 shows the magnitude of the time constant ratios for the ionosphere derived using the first ten odd harmonics of the $505 \mathrm{~Hz}$ fundamental frequency. Although there are large uncertainties on the time constant ratio, especially for the values derived from the low frequency data, most of the predicted values of time constant ratio lie between 0.75 and 1.2 .

\subsection{Even harmonics}

The information on ionospheric time constants derived for the odd harmonics of $505 \mathrm{~Hz}$, and presented in Figs. 6 and 7, can also be supplemented by data for the even harmonics. Figure 11 shows the normalised spectral response of an ionosphere with heating time constants of $2 \mu \mathrm{s}$ (upper panel) and $60 \mu \mathrm{s}$ (lower panel) when heated with an HF wave amplitude modulated at $505 \mathrm{~Hz}$. The five curves in each panel span a range of ionospheric cooling time constants from 3 to 300 times 

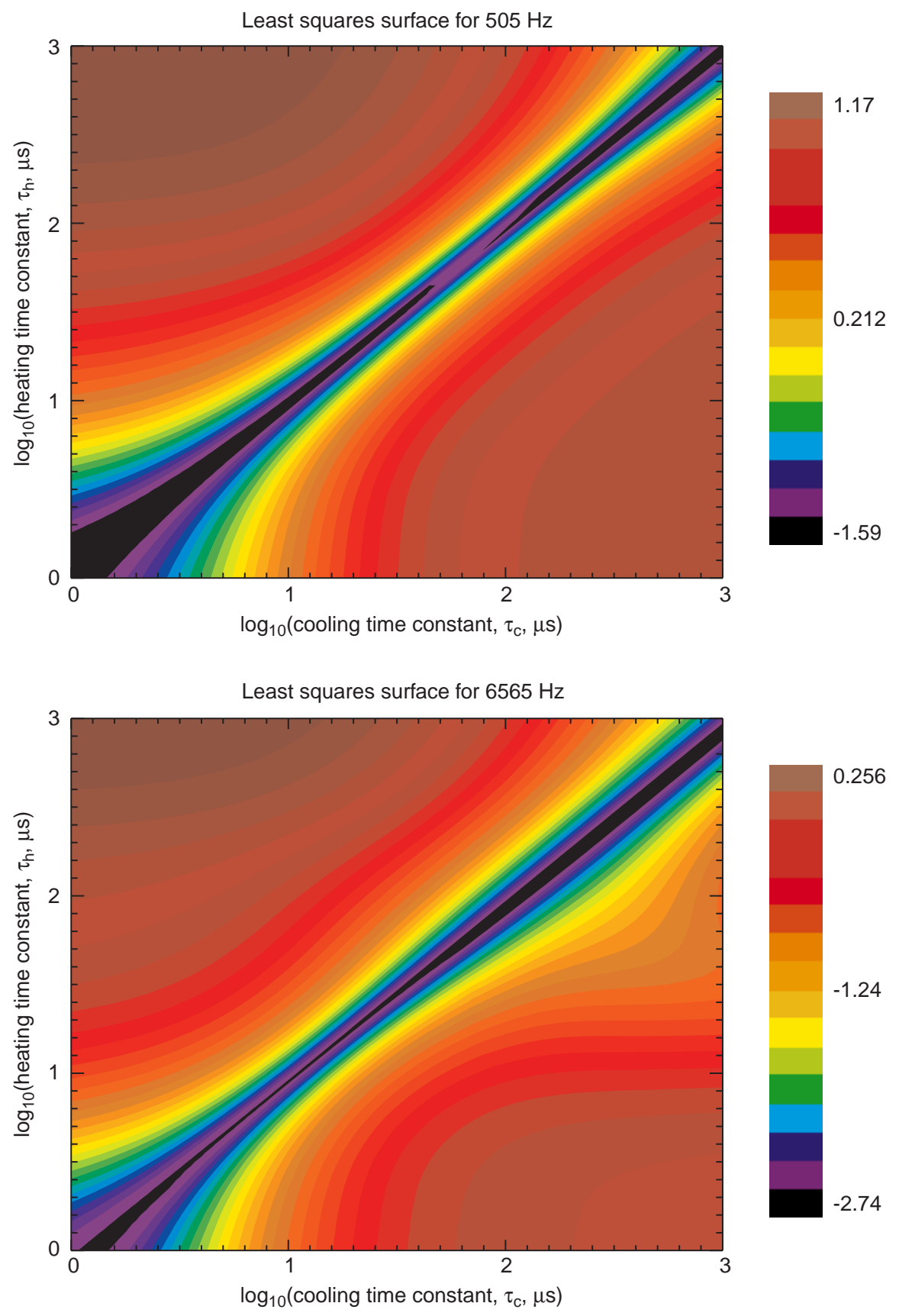

Fig. 9. Contours of $\log _{10}$ (least squares error) in fitting the experimental magnetic field strength data, measured as a function of duty cycle, to the estimates of the field strength derived theoretically from the ionospheric heating and cooling time constants. Evaluations for ODD harmonics. Upper panel: fundamental frequency, $505 \mathrm{~Hz}$. Lower panel: 13th harmonic, $6565 \mathrm{~Hz}$

the heating time constant. (Note that when the heating and cooling time constants of the ionosphere are equal no even harmonics are generated.) It is clear from Fig. 11 that if even harmonics are generated by the heating waveform then we can use them to determine the heating and cooling time constants of the ionosphere at the height at which the even harmonics are generated. Figure 12 shows the normalised spectral response for $1010 \mathrm{~Hz}$ recorded between 11:38 UT and 12:22 UT on 7 September 1990. Also shown in the same diagram are the theoretical estimates of the normalised spectral response calculated for an ionosphere with a heating time constant of $6 \mu \mathrm{s}$ and a range of cooling time constants from $18 \mu \mathrm{s}$ to $1.8 \mathrm{~ms}$. It is clear that an ionosphere with a heating time constant of $6 \mu$ s and a cooling time constant around $180 \mu$ s gives a reasonable fit to the experimental data. It should be noted that this suggests a time constant ratio $\sim 30$ for this first even harmonic compared to an average value around unity for the odd harmonics.

This is confirmed in Fig. 13 where we have plotted coloured contours of the magnitude of the error in least squares fitting the experimental ELF even harmonic spectral data to theoretically computed spectra. The theoretical computations cover ionospheric models with values of heating and cooling time constants each ranging over 3 orders of magnitude. The upper panel applies to the spectral data at $3030 \mathrm{~Hz}$ and the lower panel applies to data at $6060 \mathrm{~Hz}$. It is clear from Fig. 13 that the cooling time constants for ionospheric currents 


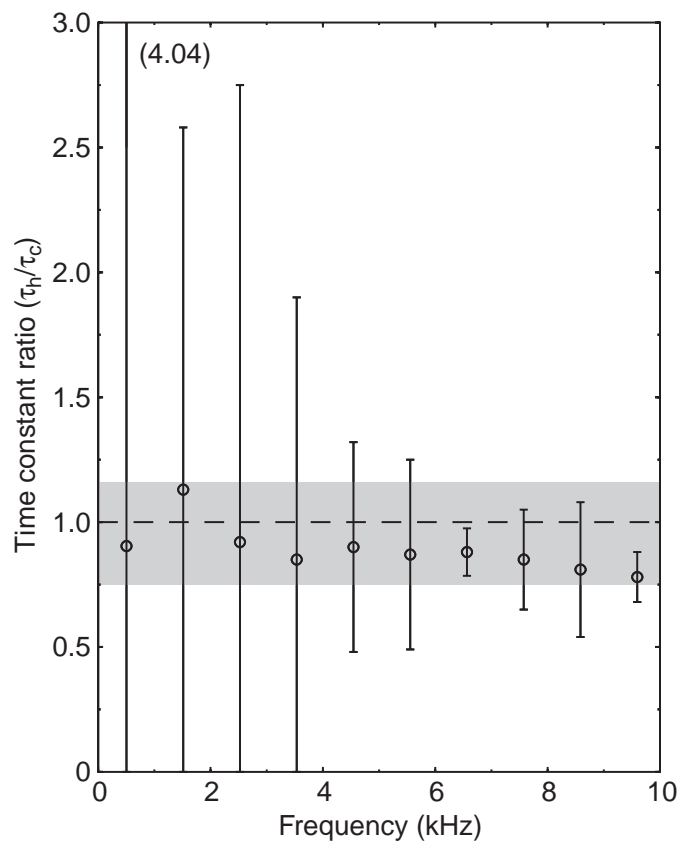

Fig. 10. Ratio of heating to cooling time constants of the modulated current in the ionosphere, derived for odd harmonics as a function of the harmonic frequency

is quite well determined but that there is significantly more uncertainty in the heating time constant. Even so it is clear that time constant ratios of around 15-30 will be
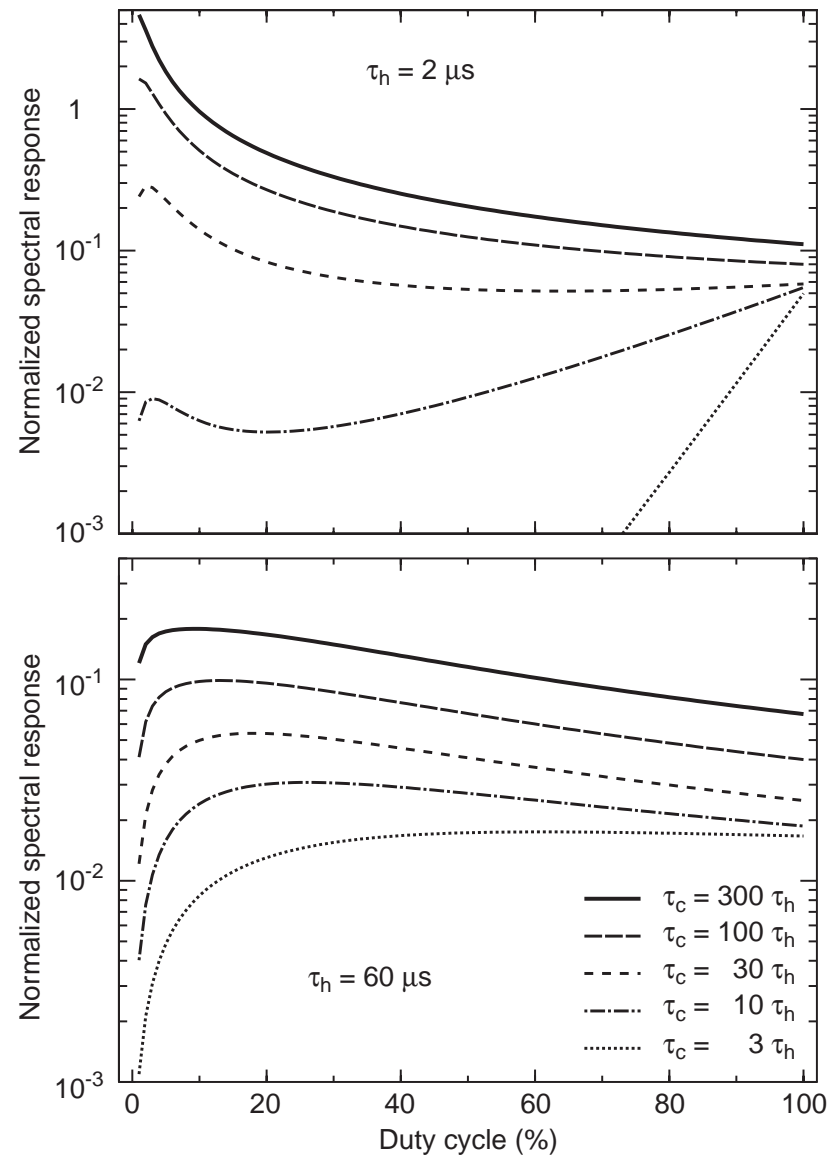

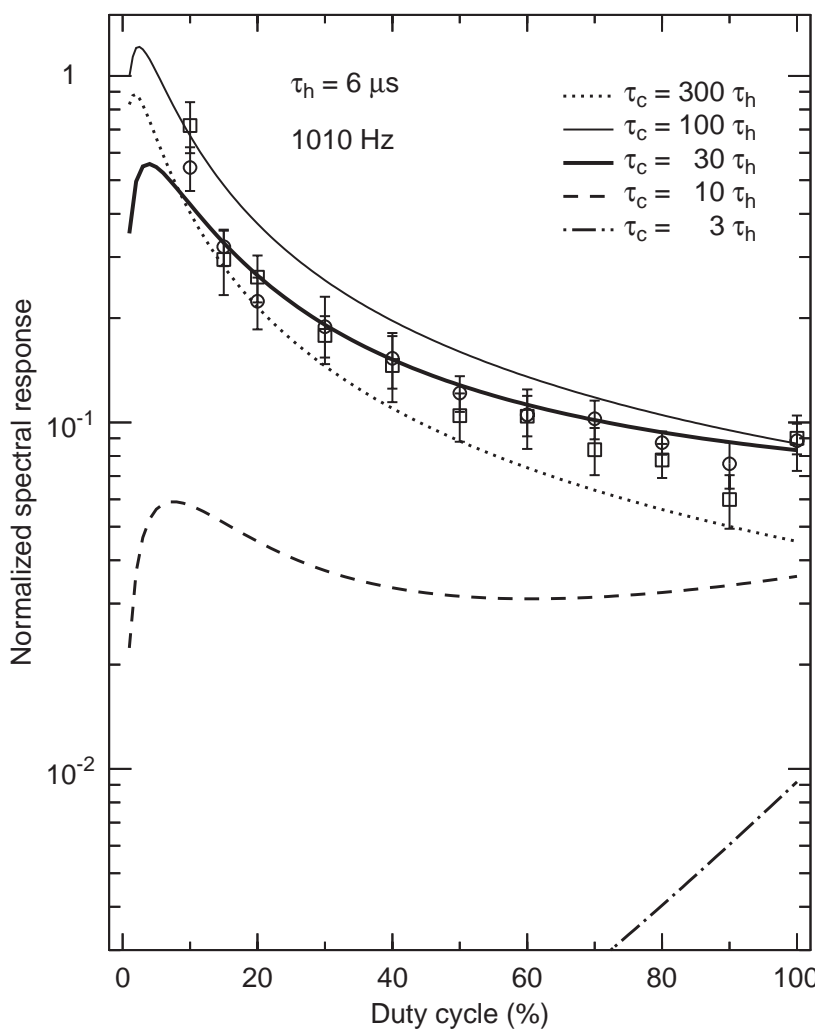

Fig. 12. Theoretical estimates of the normalised spectral response at $1010 \mathrm{~Hz}$ plotted for a heating time constant of $6 \mu$ s and a range of five cooling time constants. The square and round markers, with their associated error bars, represent the experimentally measured ' $R$ ' and ' $L$ ' wave normalised spectral responses respectively

needed to fit the experimental data. This is significantly different to the case with the odd harmonics where the time constant ratio was typically unity.

By applying the least squares program used to derive Fig. 13 it has been possible to compute the cooling time constants for the current in the ionosphere at the first nine even harmonics of the fundamental frequency of $505 \mathrm{~Hz}$. These average time constants are plotted as a function of frequency in Fig. 14 together with their standard deviations. The shaded area on Fig. 14, covering from 38 to $60 \mu \mathrm{s}$, spans the range of more than $75 \%$ of the derived cooling time constants.

\section{Discussion}

Rietveld et al. (1986) calculated the heating and cooling time constants for the Hall current flowing in a series of six auroral ionospheres when they were heated from

Fig. 11. Theoretical estimate of the normalised spectral response at $1010 \mathrm{~Hz}$, the first even harmonic of $505 \mathrm{~Hz}$, produced by modulated ionospheric currents, plotted for a range of five cooling time constants. The curves have been displaced vertically to assist comparison. Upper panel data computed for a heating time constant of $2 \mu$ s. Lower panel data computed for a heating time constant of $60 \mu \mathrm{s}$ 

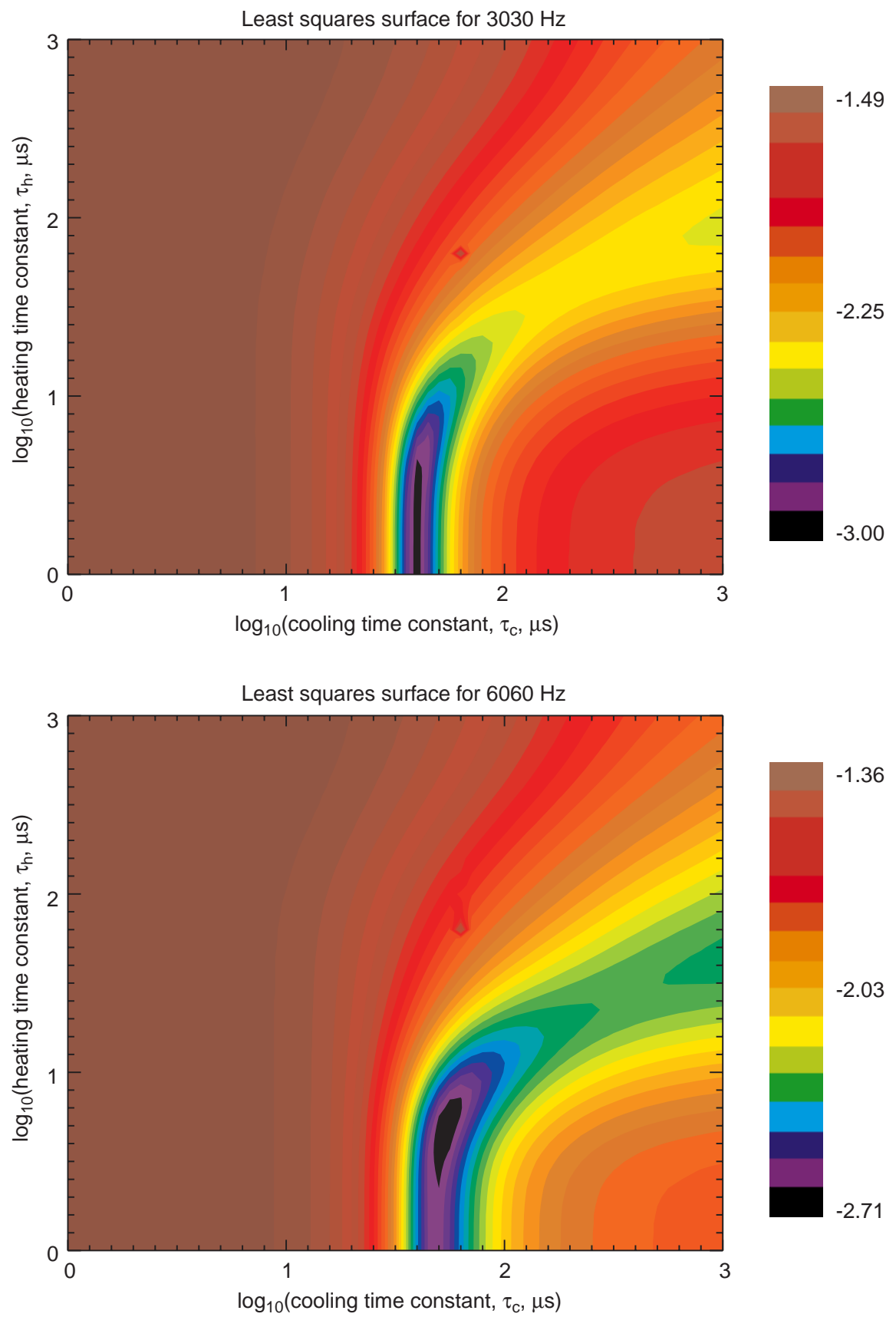

below by powerful HF waves modulated at ELF $(3 \mathrm{kHz})$. They computed the time constants for the Hall current in preference to the Pedersen current as the Hall current had been shown to produce the dominant integrated dipole moment in studies used to determine the effective excitation of the Earth-ionosphere-waveguide by ELF current sources in the ionosphere produced by amplitude modulated HF heating (Barr and Stubbe, 1984b). In Fig. 15 we present data on the Hall current cooling time constant and the Hall current time constant ratio for the ionosphere profile nr: 3 from Rietveld et al. (1986). Up to an altitude of about $85 \mathrm{~km}$ this profile is representative of a daytime ionosphere with low auroral absorption.

The small shaded rectangle plotted on the RHS of Fig. 15 has a height set by the range of time constant ratios $(0.75-1.2)$ determined to be representative of the odd harmonic data presented in Fig. 10. The width of the rectangle defines the range of ionospheric heights $\left(h^{u}-h^{l}\right)$ in which significant currents must be flowing to produce the same range of time constant ratios. We can see that if our model is correct the fundamental signal at $505 \mathrm{~Hz}$ and the odd harmonics must all be generated around $79 \mathrm{~km}$. By mapping the limiting heights $\left(h^{u}-h^{l}\right)$ upwards in Fig. 15 onto the curve for the cooling time constant $t_{c}$, we can derive the range of time constant values which our ionosphere model gives as representative of the altitude where our odd harmonic signals are generated. The cooling time lies between 160 and $170 \mu$ s and as we know that the time constant ratio is around unity these values are also representative of the heating time constant. 


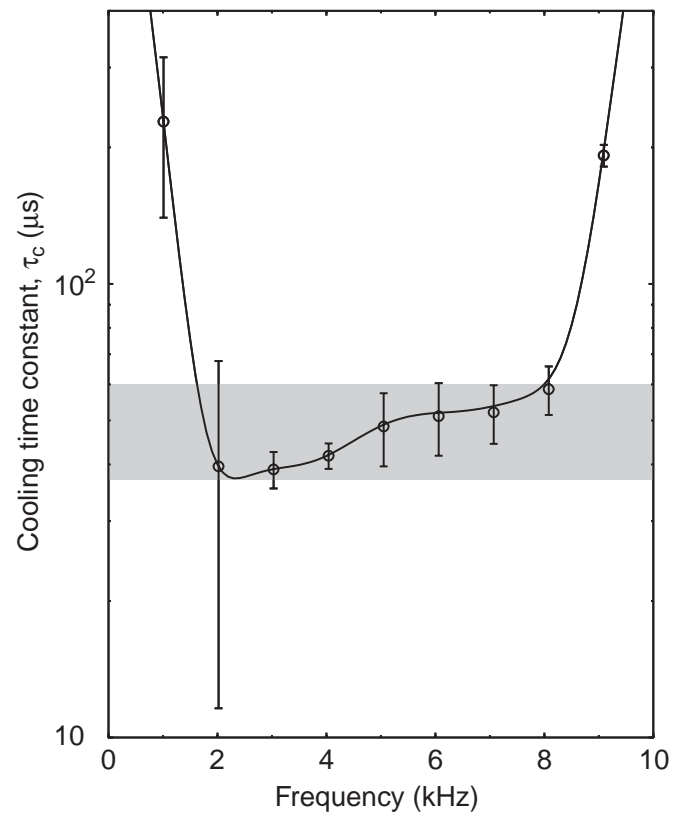

Fig. 14. Cooling time constants derived from first nine even harmonics of $505 \mathrm{~Hz}$. Grey shaded area shows range in which more than $75 \%$ of data points are accommodated

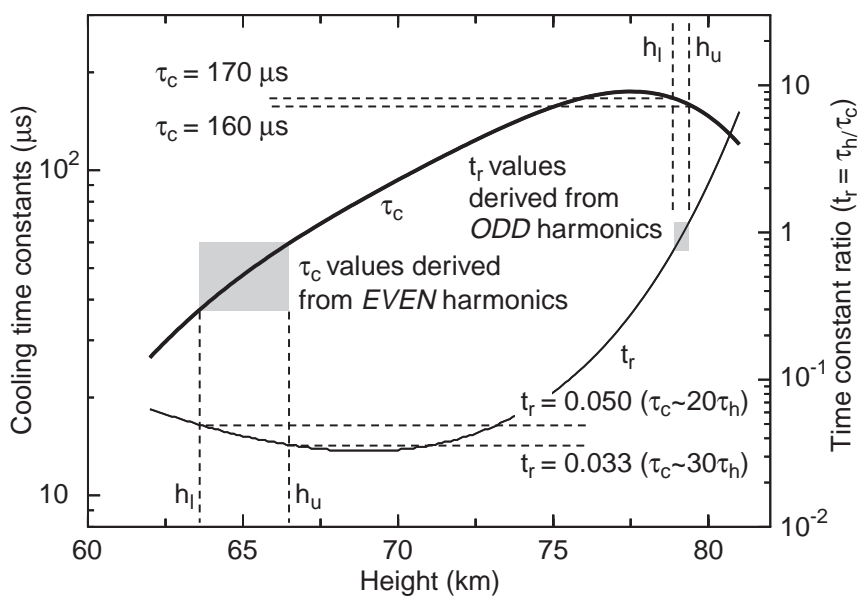

Fig. 15. Cooling time constants and time constant ratios plotted as a function of height in the ionosphere (derived from Rietveld et al. 1986)

The large shaded rectangle plotted on the LHS of Fig. 15 has a height set by the range of cooling time constants (38-60 $\mu \mathrm{s})$ determined to be representative of the even harmonic data presented in Fig. 14. The width of the rectangle defines the range of ionospheric heights $\left(h^{u}-h^{l}\right)$ in which significant currents must be flowing to produce the same range of time constant ratios. We can see that if our model is correct the even harmonics must be generated between 63 and $67 \mathrm{~km}$. By mapping these limiting heights $\left(h^{u}-h^{l}\right)$ downwards in Fig. 15 onto the curve for the time constant ratio, we can derive the range of time constant ratios which our ionosphere model gives as representative of the altitude where our even harmonic signals are generated. The model sug- gests that cooling time constants should be typically 20-30 times the heating time constant. These values are in the same range of those determined experimentally from the least square fit contours for the even harmonic data presented in Fig. 13. This therefore lends support to the theoretical models of Rietveld et al. (1986) and also to our suggestion that the even harmonics are sourced much lower in the ionosphere than the odd harmonics.

However, like all answers, once they have been discovered they evermore appear self-evident. If we send only odd harmonics into the ionosphere, even harmonics can only be generated in regions where the waveform asymmetry is changed, that is in regions where the time constant ratio is not equal to unity. As can be seen from Fig. 15 this means even harmonics must be sourced from lower altitudes than odd harmonics.

\section{Conclusion}

All the data presented here were recorded around midday in early September and the conclusions listed below can therefore be expected to be applicable to daytime operation at most high latitude heating facilities.

The effective heating and cooling time constants for the ELF Hall current in the ionosphere in a typical beam painting, pulsed HF heating scenario, depend more strongly on the nature of the harmonic being generated than on its order.

The fundamental frequency (here $505 \mathrm{~Hz}$ ) and the odd harmonics are characterised by being sourced in the ionosphere from regions supporting nearly equal heating and cooling time constants. The time constant ratio for the fundamental frequency and all odd harmonics up to the 19th is in the range 0.75-1.16. This information together with the theoretical work on heating and cooling time constants presented by Rietveld et al. (1986) allows us to estimate the source height of these frequencies at around $79 \mathrm{~km}$ in a typical daytime, high latitude ionosphere. Using the same theoretical model we estimate heating and cooling time constants to be around $165 \mu$ s.

The even harmonics are characterised by being sourced in the ionosphere from regions where heating time constants are typically 10-30 times smaller than the cooling time constants. The cooling time constants for the even harmonics lie predominantly in the range 38-60 $\mu \mathrm{s}$. This range of cooling time constants taken together with the theoretical work on heating and cooling time constants presented by Rietveld et al. (1986) enables us to estimate the source height of these frequencies as around $63-67 \mathrm{~km}$ in a typical daytime, high latitude ionosphere. Using the same theoretical model we find the time constant ratios in this altitude range lie between 20 and 30 . These values are consistent with the magnitude of the time constant ratios we derived by direct least squares fits to the data.

We therefore conclude that beam painting will not greatly enhance the magnitude of the fundamental frequency, nor the odd harmonics, of an ELF signal 
generated in the ionosphere by amplitude modulated $\mathrm{HF}$ heating at frequencies near $4 \mathrm{MHz}$ with the power levels typically used here ( $250 \mathrm{MW}$ ERP). Beam painting should, however, be effective in enhancing the magnitude of even harmonics generated by modulated HF heating at such frequencies and power levels.

These results do not preclude the possibility that by employing higher power and/or higher frequency HF heating, beam painting may also enhance the magnitude of fundamental and odd harmonic modulation frequencies.

Acknowledgements. We thank Helmut Gegner for his efficient operation and maintenance of the heater facility during this study and also for his help with the ELF field measurements. The Heating facility was supported by the Deutsche Forschungsgemeinschaft (DFG) and the Max-Planck-Gesellschaft (MPG) and operated by the Max-Planck-Institut für Aeronomie (MPAe). It is currently supported, through the EISCAT Scientific Association, by the research councils of Finland, France, Germany, Japan, Norway, Sweden and UK.

Topical Editor D. Alcaydé thanks K. Papadopoulos and K. J. Harker for their help in evaluating this paper.

\section{References}

Barr, R., and P. Stubbe, The 'Polar Electrojet Antenna' as a source of ELF radiation in the Earth-ionosphere waveguide, $J$. Atmos. Terr. Phys., 46, 315-320, 1984a.

Barr, R., and P. Stubbe, ELF and VLF Radiation from the Polar Electrojet Antenna, Radio Sci., 19, 1111-1122, 1984 b.

Barr, R., and P. Stubbe, ELF radiation from the Tromsø 'Super Heater' facility, Geophys. Res. Lett., 18, 1035-1038, 1991.

Barr, R., and P. Stubbe, ELF harmonic radiation from the Troms heating facility, Geophys. Res. Lett., 20, 2243-2246, 1993.

Barr, R., M. T. Rietveld, H. Kopka, P. Stubbe, and E. Nielsen, Extra-low-frequency radiation from the polar electrojet antenna, Nature, 317, 155-157, 1985.

Barr, R., P. Stubbe, M. T. Rietveld, and E. Nielsen, Enhanced ELF wave generation efficiency using 'O' mode HF heating, Geophys. Res. Lett., 24, 1403-1406, 1997.

Budden, K. G., Radio waves in the ionosphere, Cambridge University Press, London, 1966.

Burrows, M. L., ELF Communications Antennas, Peter Peregrinus, Stevenage, UK, 1978.

Getmantsev, G. G., $\quad$ N. A. Zuikov, D. S. Kotik, L. F. Mironenko, N. A. Mityakov, V. A. Rapoport, Yu. A. Sazonov,
V. Yu. Trakhtengerts, and V. Ya. Eidman, Combination frequencies in the interaction between high-power short-wave radiation and ionospheric plasma, JETP Lett. (Engl. Transl.), 20, 101-102, 1974.

Kapustin, I. N., R. A. Pertsovskii, A. N. Vasilev, V. S. Smirnov, O. M. Raspopov, L. E. Solov'eva, A. A. Ul'yachenko, A. A. Arykov, and N. V. Galakhova, Generation of radiation at combination frequencies in the region of the auroral electric jet, JETP Lett. (Engl. Transl.), 25, 228-231, 1977.

Kotik, D. S., and V. Yu. Trakhtengerts, Mechanism of excitation of combination frequencies in ionospheric plasma, JETP Lett. (Engl. Transl.), 21, 51-52, 1975.

McCarrick, M. J., A. Y. Wong, R. F. Wuerker, B. Chouinard, and D. D. Sentman, Excitation of ELF waves in the Schumann resonance range by modulated $\mathrm{HF}$ heating of the polar electrojet, Radio Sci., 25, 1291-1298, 1990.

Papadopoulos, K., A. S. Sharma, and C. L. Chang, On the efficient operation of a plasma ELF antenna driven by modulation of ionospheric currents, Comments Plasma Phys. Controlled Fusion, 13, 1, 1989.

Papadopoulos, K., C. L. Chang, P. Vitello, and A. Drobot, On the efficiency of ionospheric ELF generation, Radio Sci., 25, 13111320, 1990.

Rietveld, M. T., H. Kopka, E. Nielsen, P. Stubbe, and R. L. Dowden, Ionospheric electric field pulsations: a comparison between VLF results from an ionospheric heating experiment and STARE, J. Geophys. Res., 88, 2140-2146, 1983.

Rietveld, M. T., R. Barr, H. Kopka, E. Nielsen, P. Stubbe, and R. L. Dowden, Ionospheric heater beam scanning: a new technique for ELF studies of the auroral ionosphere, Radio Sci., 19, 1069-1077, 1984.

Rietveld, M. T., H. Kopka, and P. Stubbe, D-Region characteristics deduced from pulsed ionospheric heating under auroral electrojet conditions, J. Atmos. Terr. Phys., 48, 311-326, 1986.

Rietveld, M. T., P. Stubbe, and H. Kopka, On the frequency dependence of ELF/VLF waves produced by modulated ionospheric heating, Radio Sci., 24, 270-278, 1989.

Rietveld, M. T., H. Kohl, H. Kopka, and P. Stubbe, Introduction to ionospheric heating at Tromsø I. Experimental overview, J. Atmos. Terr. Phys., 55, 577-599, 1993.

Stubbe, P., H. Kopka, and R. L. Dowden, Generation of ELF and VLF waves by the polar electrojet modulation: experimental results, J. Geophys. Res., 86, 9073-9078, 1981.

Stubbe, P., H. Kopka, M. T. Rietveld, and R. L. Dowden, ELF and VLF wave generation by modulated HF heating of the current carrying lower ionosphere, J. Atmos. Terr. Phys., 44, 1123$1135,1982$.

Willis, J. W., and J. R. Davis, Radio frequency heating effects on the electron density in the lower E region, J. Geophys. Res., 78, 5710-5717, 1973. 